\title{
Estimating the Effects of Developing Wrong Software Functions using Fuzzy Cognitive Map
}

\author{
Ramandeep Kaur, \\ Research Scholar, \\ Lovely Professional University, \\ Phagwara (Punjab) India
}

\author{
Neha Malhotra \\ Assistant Professor, \\ Lovely Professional University, \\ Phagwara (Punjab) Indin
}

\begin{abstract}
Risk management is an important area in the field of Software Engineering. In software development industry there are many types of risks which can leads to project failure. From these risks functionality risk is one that needs to be focus for software development industry. Due to this risk many problems can arise. If effects of this risk are not estimated it would pose problems for the success of the project. By estimating the effects of developing wrong software functions developer will come to know about the problems they have to face with the occurrence of this risk and considering these effects developer can take appropriate decisions to manage this risk. So, we have designed a FCM based tool to estimate the effects of developing wrong software functions. The developed tool will be helpful for developers to estimate the effects of developing wrong software functions on the success of project and would be helpful for providing awareness of consequences of this risk.
\end{abstract}

\section{Keywords}

Risk management, Fuzzy Cognitive map, Fuzzy Inference System.

\section{INTRODUCTION}

Software engineering is the process of solving customer's problems by the systematic development and evolution of large, high quality software systems within cost, time and other constraints [17]. Due to the complex of software development industry, there can be unexpected problems that results in project delay, exceeding budget and quality problems related to products. Neglecting those risks will results in a project failure or poor quality software that partially fulfills the user requirement and thus leads to the dissatisfaction of user. Although such problems or risks cannot be totally eliminated from software projects but can be managed. Risk management can help to deal with problems before they occur. Risk management is a way for managing risks and concerns with all activities that are performed to reduce uncertainty associated with certain tasks or event [15].

\section{RISK OF DEVELOPING WRONG SOFTWARE FUNCTIONS}

From the software risk items listed in [1] [3] [12] developing wrong software functions is one. The function of the software is to perform the work that the user needs from the software to do. Functionality risk is defined as the risk that a completed system will not meet its users' needs [4]. If the developer develops the wrong software function then various problems can arise due to this like incorrect operation of the software, unexpected result, increase in maintenance for taking corrective actions, dissatisfaction of user, overhead of rework, product rejection, adversely affecting developer reputation and many other problems can arise that can become a challenge for software project and can lead the project to the failure. To estimate the effects of developing wrong software functions we have used the approach of fuzzy cognitive map [15].

\section{FUZZY COGNITIVE MAP}

Fuzzy Cognitive Map (FCM) is a cognitive map in which relations between elements like events, concepts, project resources can be used to compute the "strength of impact" of these elements. FCM have been introduced by Kosko (1986) as signed directed graph for representing casual reasoning and computational inference processing, exploiting a symbolic representation for the description and modeling of complex system. Fuzzy cognitive map defines different aspects of the behavior of complex system in terms of concepts. The construction of a FCM requires the input of human experience and knowledge on the system under consideration [6]. The graphical representation of FCM consists nodes and directed edges. Nodes represent the concepts that are used to describe the behavior of the system, connected by signed and weighted edges that represents the casual relationship that exist between concepts i.e the influence of cause variable on effect variable [15].

\section{RELATED WORK}

This section discusses the use of Fuzzy cognitive map ( FCM) approach in different scenarios. A tool based on FCM is built that helps ERP teams to simulate the impact of risks on ERP maintenance.FCM is constructed for the identification of ERP maintenance risks and the existing connections or relations between them [2]. Fuzzy logic based decision support system is developed which helps to estimate the risk associated with the software development team [7]. From the various factors that can lead to project failure gold plating is one. For estimating the effects of gold plating a tool based on fuzzy cognitive maps is designed. This tool can help developer to know the effects of doing gold plating on the project [8]. Dynamic simulation tool is built that allows ERP manager to foresee the impact of risks on maintenance goal. To do this augmented FCM approach is used. The result proves that risks strongly effect ERP maintenance goals [9]. There are many factors that affect the quality of software. These factors can cause various problems in the projects. Decision support system is proposed to analyze software risks [10]. The approach of FCM is applied to show the positive and negative casual relationship between the factors which influence the Smartphone user's overall satisfaction. Factors affecting customer satisfaction from Smartphone are investigated and 
identification is done how these factors relate to each other [11].

\section{PROPOSED WORK}

The proposed tool to estimate the effects of developing wrong software functions on project failure is based on fuzzy cognitive map. This tool calculates the effects of input factors on output factors. Number of factors have been find out which can arise if wrong software functions are developed by the developer. After finding these factors, interdependencies between these factors have been determined. Experts' views have been taken to know which factor have how much effect level on output factors through filling up survey form. After analyzing experts opinions 15 input factors are selected to make the tool and with the help of this tool the effects of selected input factors on 3 output factors is calculated. Input factors are shown in table below.

Table 5.1

\begin{tabular}{|c|l|}
\hline S.no & \multicolumn{1}{|c|}{ Input Factors } \\
\hline 1. & Incorrect operation of the software \\
\hline 2. & Unexpected Result \\
\hline 3. & Missing out required functionality \\
\hline 4. & Complexity \\
\hline 5. & Poor quality product \\
\hline 6. & Dissatisfaction of user \\
\hline 7. & Product rejection \\
\hline 8. & Undermined motivation \\
\hline 9. & Friction between developer and customer \\
\hline 10. & Increase in maintenance for taking corrective action \\
\hline 11. & Overhead of rework \\
\hline 12. & Extra cost of maintenance \\
\hline 13. & Extra time for maintenance \\
\hline 14. & Work loss of user \\
\hline 15. & Adversely affecting developer reputation \\
\hline
\end{tabular}

The effect of selected input factors on 3 output factors is checked. Three output factors are shown in table below.

\section{Table 5.2}

\begin{tabular}{|l|l|}
\hline S.no & \multicolumn{1}{|c|}{ Output Factors } \\
\hline 1. & Chances of Technical Difficulties \\
\hline 2. & Chances of Project Failure \\
\hline 3. & Chances of Decrease in Quality Of Software \\
\hline
\end{tabular}

The construction of rule base is done using 30 different rules which are designed on the basis of interdependencies among the factors. In order to estimate the effects of developing wrong software functions on project failure, FCM based GUI tool is developed in MATLAB on the basis of 30 rules. The GUI tool that we have built calculates the effects of selected input factors in percentage on 3 output factors.

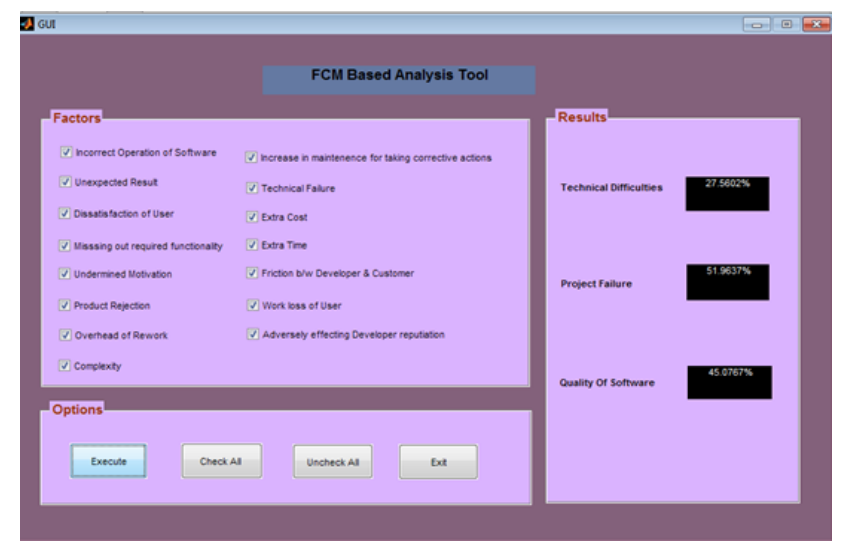

Fig 1: GUI Tool Based on the Fuzzy Cognitive Map

\subsection{Methodology of Design}

Five different ranges of effect levels are considered i.e. "Very Low", "Low", "Medium", "High", and "Very High" for estimating the effects. To design fuzzy system inputs are represented by fuzzy sets. Membership functions are used to represent fuzzy sets. Different types of membership functions are used for designing the fuzzy inference system in MATLAB. Triangular-shaped built-in membership functions are used for input factors. Triangular-shaped and trapezoidalshaped membership functions are used for output factors. The triangular-shaped membership function is specified by three parameters $\{a, b, c\}$ as follows in e.q 1 .

$$
\text { Triangle }(\mathrm{x}: \mathrm{a}, \mathrm{b}, \mathrm{c})=\begin{array}{lc}
0 & \mathrm{x}<\mathrm{a} \\
(\mathrm{x}-\mathrm{a}) /(\mathrm{b}-\mathrm{a}) & \mathrm{a}<=\mathrm{x}<=\mathrm{b} \\
(\mathrm{c}-\mathrm{x}) /(\mathrm{c}-\mathrm{b}) & \mathrm{b}<=\mathrm{x}<=\mathrm{c} \\
0 & \mathrm{x}>\mathrm{c}
\end{array}
$$

Using IF-THEN rules of fuzzy logic, relationships among factors is defined. Inputs and outputs of fuzzy sets are represented in membership functions and after that rules are formulated. For example, In the case of Friction b/w Developer \& Customer which can lead to Project Failure, its rule is as given as:

If (FrictionB/WDeveloper\&Customer is YES) then (ProjectFailure is H) (0.75)

If (FrictionbBWDeveloper\&Customer is YES) then (ProjectFailure is M) (0.25)

In the case of Technical Failure which can lead to Technical Difficulties, its rule is as given as:

If(TechnicalFailure is YES) then (TechnicalDifficulties is M) $(0.25)$ 
If(TechnicalFailure is YES) then (TechnicalDifficulties is $\mathrm{H}$ ) (0.75)

\subsection{Implementation}

For implementation of research methodology firstly fuzzy inference system is designed that is the process through which we can formulate the mapping from a given input to an output using fuzzy logic. On the basis of this mapping we can make decision. In this work mamdani type FIS system is used. Fuzzy inference system showing the mapping of inputs and outputs is given in Figure 2.

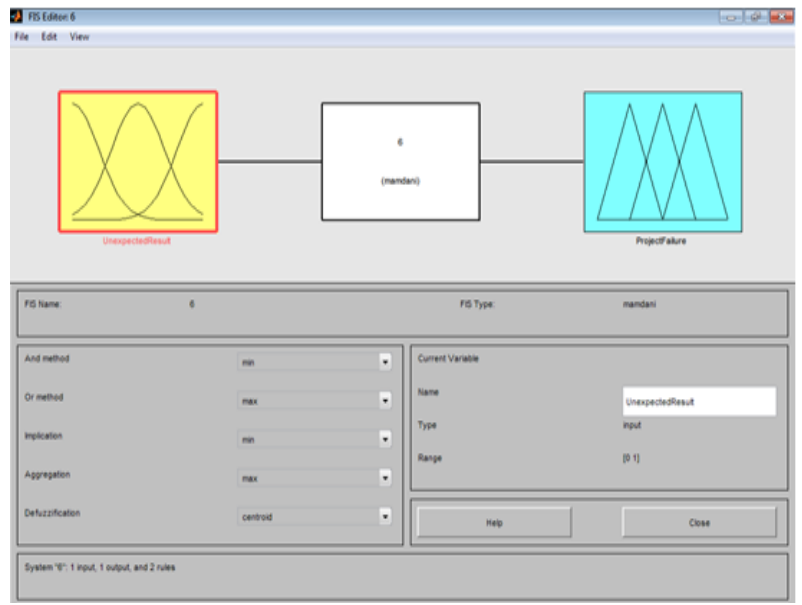

Fig 2: Fuzzy inference system showing the mapping of inputs and outputs

In Rule editor 30 different rules are constructed which shows that if particular input factor exist what would be the effect of that input factor on output factor i.e "Very Low", "Low", "Medium", "High", and "Very High". Rule editor provides facility to delete, add and change rule. Rule editor is shown in Figure 3.

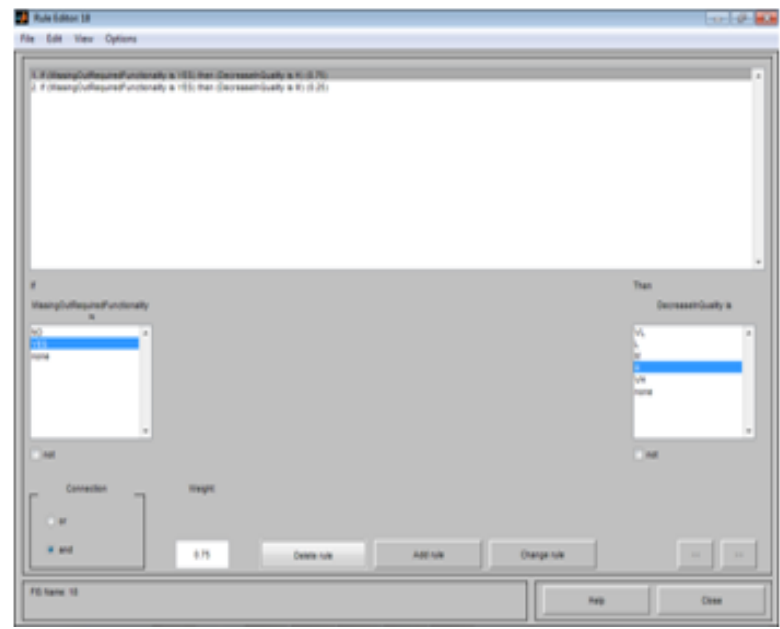

Fig 3: Rule Editor

Rule viewer computes the interdependencies among the various dependent factors and with the help of rule viewer weights of dependent factors are calculated. Rule viewer is shown in Figure 4.

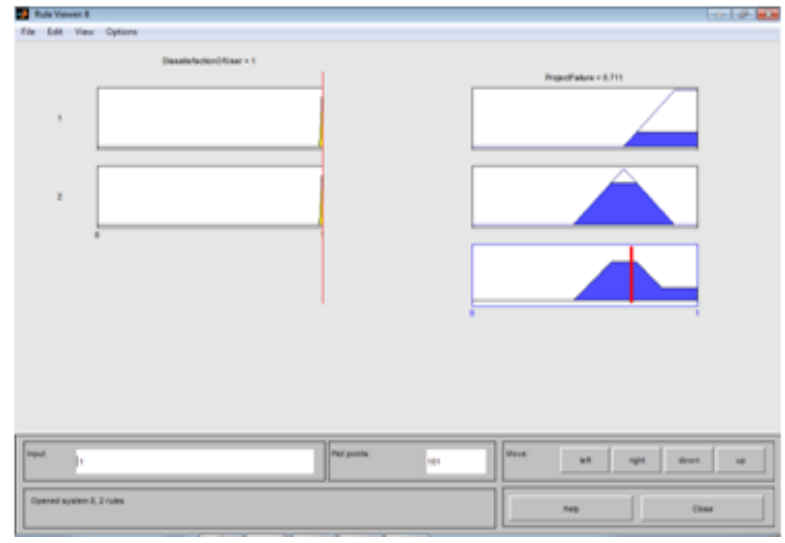

Fig 4: Rule Viewer

Surface view shows us the output surface of Fuzzy Inference system for any input. Surface view is shown in Figure 5.

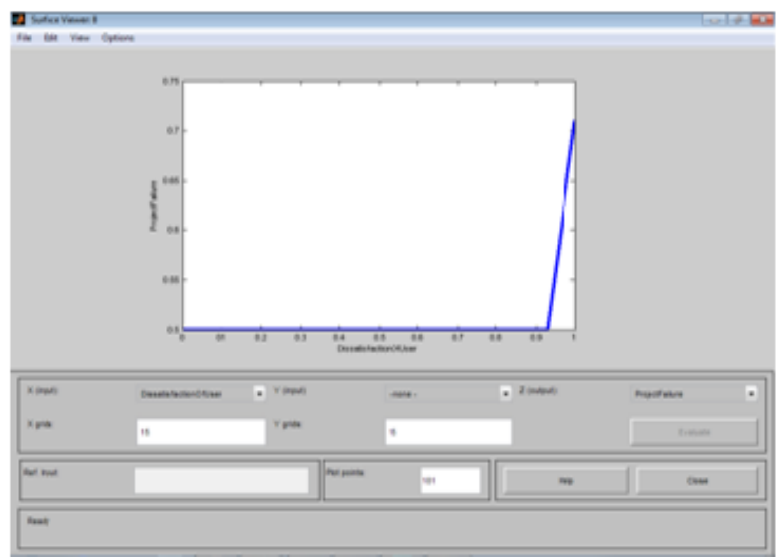

Fig 5: Surface Viewer

The Figure 6. represents the tool showing the impact on 3 output factors when certain input factors like Incorrect operation of software, Unexpected result, Missing out required functionality, Product Rejection , Overhead of rework, Increase in maintenance for taking corrective action, Extra Cost, Friction b/w developer \& customer. The tool calculates percentage of effect on the output factors due to the input factors.

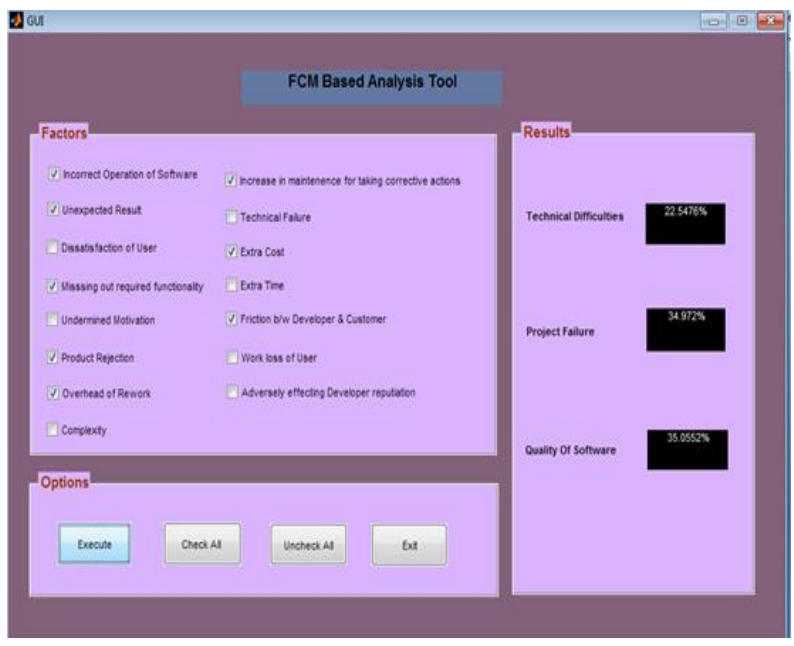

Fig 6: GUI tool showing output 


\section{CONCLUSION}

Risk management is an important research area in software engineering. Risks can be tackled more efficiently with the proper awareness about the consequences of particular risk. Developing wrong software functions is one of major risk in software risk items. This paper proposed a tool which will help the developer to estimate the effects of developing wrong software functions on the project and to make appropriate decisions regarding the project based on the output produced by the tool. In future, proposed system can be more efficient with identifying more factors of this risk and analyzing their effects.

\section{REFERENCES}

[1] Boehm B. W, "Software Risk Management: Principles and Practices." IEEE Software, January 1991, pp. 3241.

[2] Cristina Lopez, Jose L. Salmeron, Sebastian Lozano" Software Maintenance Scenarios Simulation with Fuzzy Cognitive Maps" IEEE International Conference on Fuzzy Systems June 27-30, 2011, Taipei, Taiwan.

[3] Janne Ropponen and Kalle Lyytinen" Components of Software Development Risk: How to Address Them? A Project Manager Survey" IEEE TRANSACTIONS ON SOFTWARE ENGINEERING, VOL. 26, NO. 2, FEBRUARY 2000.

[4] Amrit Tiwana and Mark Keil, Member, IEEE” Functionality Risk in Information Systems Development: An Empirical Investigation" IEEE TRANSACTIONS ON ENGINEERING MANAGEMENT, VOL. 53, NO. 3, AUGUST 2006.

[5] C. E. Pelaez and J. B. Bowles, "Using fuzzy Cognitive Maps as a System Model for Failure Models and Effects Analysis'Information Sciences, Vol. 88, pp. 177-199, 1996.

[6] Elpiniki Papageorgiou and Areti Kontogianni"Using Fuzzy Cognitive Mapping in Environmental Decision Making and Management: A Methodological Primer and an Application" International Perspectives on Global Environmental Change.

[7] Simranjeet Kaur, Ms. Raman Goyal "Fuzzy Logic Based Decision Support System for Poor Team Cohesiveness" "International Journal for Science and Emerging Technologies with Latest Trends" 2(1): 1-6 (2012).
[8] Neha Malhotra ,Manisha Bhardwaj and Rajwinder Kaur "Estimating the Effects of Gold Plating Using Fuzzy Cognitive Maps" International Journal of Computer Science and Information Technologies, Vol. 3 (4), 2012.

[9] Jose L. Salmeron and Cristina Lopez "Forecasting Risk Impact on ERP Maintenance with Augmented Fuzzy Cognitive Maps "IEEE TRANSACTIONS ON SOFTWARE ENGINEERING, VOL 38, NO.2 MARCH/APRIL2012.

[10] Surbhi Anand and Vinay Chopra" Decision Support System for Software Risk Analysis during Software Development "International Journal for Science and Emerging Technologies with Latest Trends" 2(1): 29-35 (2012)

[11] Gerogiannis,V. C., Papadopoulou and S. Papageorgiou, E. I." Identifying Factors of Customer Satisfaction from Smartphones: A Fuzzy Cognitive Map Approach “ International Confereneeringnce on Contemporary Marketing Issues (ICCMI) 2012

[12] Rashmita Das and Rajashree Das "Risk Assessment Techniques for Software Development" European Journal of Scientific Research ISSN 1450-216X Vol.42 No.4 (2010), pp.629-636.

[13] G.N.K. Suresh Babu1 and S.K. Srivatsa" Increasing Success of Software Projects through Minimizing Risks" International Journal of Research and Reviews in Software Engineering Vol. 1, No. 1, March 2011.

[14] Namrata kapoor, Nitin Bhatia, Sangeet Kumar, "Software Risk Analysis Using Fuzzy Logic" International Journal of Computer Information Systems, Vol. 2, No.2 , 2011.

[15] Ramandeep Kaur, Neha Malhotra "A Method for Estimating the Effects of Functionality Risk"International Journal of Engineering and Advanced Technology (IJEAT) ISSN: 2249 - 8958, Volume-2, Issue-3, February 2013

[16] W.R. Zhang, S. S. Chen, W. Wang and R. S. King, 'A Cognitive-Map-Based approach to the coordination of distributed cooperative agents' IEEE Transactions onSystems, Man, and Cybernetics, Vol. 22, No1, pp. 103-114, 1992.

[17] Timothy C.Lethbridge "Object oriented software Engineering practical software development using UML and java" Tata McGraw Hill Education Private Limited. 